\title{
Experimentally Verified Generalized KYP Lemma based Iterative Learning Control Design ${ }^{\text {负 }}$
}

\author{
Wojciech Paszke $^{\mathrm{a}, *}$, Eric Rogers ${ }^{\mathrm{b}}$, Krzysztof Gałkowski ${ }^{\mathrm{a}}$ \\ ${ }^{a}$ University of Zielona Góra, ul. Szafrana 2, 65-516 Zielona Góra, Poland, \\ Phone: +48 683283219, Fax: +48683284751 \\ ${ }^{b}$ Electronics and Computer Science, University of Southampton, Southampton SO17 1BJ, United Kingdom
}

\begin{abstract}
This paper considers iterative learning control law design for plants modeled by discrete linear dynamics using repetitive process stability theory. The resulting one step linear matrix inequality based design produces a stabilizing feedback controller in the time domain and a feedforward controller that guarantees convergence in the trial-to-trial domain. Additionally, application of the generalized Kalman-Yakubovich-Popov (KYP) lemma allows a direct treatment of differing finite frequency range performance specifications. The results are also extended to plants with relative degree greater than unity. To support the algorithm development, the results from an experimental implementation are given, where the performance requirements include specifications over various finite frequency ranges.
\end{abstract}

Keywords: Iterative learning control, finite frequency range design, the generalized KYP lemma, experimental verification.

\section{Introduction}

Iterative learning control (ILC) has been especially developed for systems defined over a finite duration that repeat the same task. Each execution is known as a trial, or pass, and the sequence of operations is that a trial is completed, the system resets to the starting location and then the next trial begins, either immediately after the resetting is complete or a period of time has elapsed since completion of the resetting. The novel feature of this control law design method is the use information from the previous trial, or a finite number of previous trials, to update the control input applied on the next trial and thereby improve performance from trial-to-trial. In particular, the control objective is to find a control input such that the corresponding output precisely tracks a reference signal that is specified over a finite time interval.

Since the original work, widely credited to (Arimoto et al., 1984), ILC has remained as a significant area of control systems research with, especially for linear model based designs, many algorithms experimentally verified in the research laboratory and applied in industrial and other applications. An overview of developments up to their dates of publication can be found in, e.g., the survey papers (Ahn et al., 2007; Bristow et al., 2006; Wang et al., 2009), where the last of these has a particular focus on run-to-run control as found in the chemical process industries. Applications areas include industrial robotics, see, e.g., (Longman, 2000; Norrlöf, 2002), where the pick and place operation common in

\footnotetext{
This work is partially supported by National Science Centre in Poland, grant No. 2014/15/B/ST7/03208

* Corresponding author

Email addresses: w.paszke@issi.uz.zgora.pl (Wojciech Paszke), etar@ecs.soton.ac.uk (Eric Rogers), k.galkowski@issi.uz.zgora.pl (Krzysztof Gałkowski)
} 
many mass manufacturing processes is an immediate fit to ILC, and wafer stage motion systems, see, for example, (Heertjes and Tso, 2007). More recent applications areas include flexible valve actuation for non-throttled engine load control (Heinzen et al., 2011) and various forms of industrial printing, see, for example, (Barton et al., 2011; Bolder, 2015) and large dynamic range nanoprinting (Parmar et al., 2014). Also there has been a transfer from engineering to next generation healthcare for robotic-assisted upper limb stroke rehabilitation with supporting clinical trials (Freeman et al., 2009, 2012).

One common approach, see, for example, (Ahn et al., 2007; Bristow et al., 2006) as starting points for the literature, to ILC design is to first apply a feedback control law to stabilize and/or produce acceptable along the trial dynamics and then apply ILC to force trial-to-trial error convergence of the resulting system. This is a two step design approach with separate design of the feedback and learning filters where, for example, the ILC learning update is calculated as the inverse of the dynamics resulting from the feedback controller design. Moreover, the ILC learning update is a feedforward signal from the previous trial and hence does not affect the stability property of the dynamics along the trials, that is, as the trial duration is finite, trial-to-trial error convergence can occur for unstable dynamics but the result could be unsatisfacotry/unacceptable along the trial dynamics. This is a two-stage design and for discrete dynamics using the lifting approach can result in the need for computation with very large dimensioned matrices.

An alternative approach to ILC design is to use the 2D systems setting, i.e., systems that propagate information in two independent directions where for ILC these directions are from trial-to-trial and along each trial respectively. Early work in this setting used the Roesser state-space model for 2D discrete linear systems (Kurek and Zaremba, 1993; Paszke et al., 2007). Repetitive processes (Rogers et al., 2007) are a distinct class of $2 \mathrm{D}$ systems where information in the temporal domain is limited to a finite duration and hence a more natural match to ILC. These processes make a series of sweeps, termed passes or trials in the ILC setting, through a set of dynamics defined over a finite duration and once each pass is complete the process resets to the starting location. On each pass and output, termed the pass profile, is produced that acts as a forcing function on, and hence contributes to, the dynamics of the next pass profile. The result can be oscillations that increase in amplitude from pass-to-pass.

Repetitive processes cannot be controlled by direct application of standard systems theory and algorithms and this has led to the development of a stability theory for them and substantial progress on control system specification and design (Rogers et al., 2007). More recent research has used the repetitive process setting to design ILC laws with experimental verification (Hładowski et al., 2010, 2012). The result is a one step design for trial-to-trial error convergence and transient response along the trials and hence simultaneous treatment of the trial-to-trial error and transient response along the trials is possible.

The new design developed in this paper considers simultaneous synthesis of both feedback and learning controllers in an ILC scheme for error convergence and performance, starting with a new result for monotonic trial-to-trial error convergence. This result is achieved by converting the problem to one of stability along the trial for a discrete linear repetitive process, leading to design based on Linear Matrix Inequality (LMI) computations. Also the benefits of this new result relative to existing alternatives are highlighted.

As in other areas for linear systems theory and design, it is necessary to design for stability and performance, where for the latter aspect the requirements for each case must be formulated into design constraints. Such specifications can include regulating against the effects of exogenous signals, penalizing regulated variables and specifying the amount of plant uncertainty allowed. In this paper it is established that, as in other areas of standard linear systems analysis and design, such requirements can be expressed as conditions on the maximum singular value of the frequency 
response matrix coupling the errors on successive trials.

One method, again as in standard case, would be to introduce weighting filters to emphasize a particular frequency range, followed by design to ensure that weighted system norm is sufficiently small. In this paper, the generalized KYP lemma is use to develop a new design method where weighting filters are not required and hence an unnecessary increase in the controller order is avoided. To support the analysis and design, results from experimental application to an electromechanical system are given. Another contribution of the paper is the development of a method deal with the case when the system to be controlled has relative degree greater than unity through the use of an anticipative feedforward control law.

Throughout this paper, the null and identity matrices with the required dimensions are denoted by 0 and $I$, respectively, and the notation $X \prec Y$ (respectively $X \succ Y$ ) means that the matrix $X-Y$ is negative definite (respectively, positive definite). Also $\operatorname{sym}\{M\}$ is used to denote the symmetric matrix $M+M^{\top}$ and $\rho(\cdot)$ denotes the spectral radius of its matrix argument, i.e., if $\lambda_{i}, 1 \leq i \leq q$, denotes the eigenvalues of a $q \times q$ matrix, say $H, \rho(H)=\max _{1 \leq i \leq q}\left|\lambda_{i}\right|$. The superscript $*$ denotes the complex conjugate transpose of a matrix and $\otimes$ the matrix Kronecker product.

Use will also be made of the following results, where the first is the generalized KYP lemma and the second the Elimination (or Projection) Lemma.

Lemma 1. (Iwasaki and Hara, 2005) Consider matrices $\mathbb{A}, \mathbb{B}_{0}, \Theta$ and

$$
\Phi=\left[\begin{array}{cc}
-1 & 0 \\
0 & 1
\end{array}\right], \Psi=\left[\begin{array}{cc}
0 & \mathrm{e}^{\mathrm{j} \omega_{c}} \\
\mathrm{e}^{-\mathrm{j} \omega_{c}} & -2 \cos \left(\omega_{d}\right)
\end{array}\right]
$$

with $\omega_{c}=\left(\omega_{l}+\omega_{u}\right) / 2, \omega_{d}=\left(\omega_{u}-\omega_{l}\right) / 2$ and $\omega_{l}, \omega_{u}$ satisfying $-\pi \leq \omega_{l} \leq \omega_{u} \leq \pi$. Suppose also that $\operatorname{det}\left(\mathrm{e}^{\mathrm{j} \omega} I-\mathbb{A}\right) \neq 0$ for all $\omega \in\left[\omega_{l}, \omega_{u}\right]$. Then the following statements are equivalent.

i)

$$
\left[\begin{array}{c}
\left(\mathrm{e}^{\mathrm{j} \omega} I-\mathbb{A}\right)^{-1} \mathbb{B}_{0} \\
I
\end{array}\right]^{*} \Theta\left[\begin{array}{c}
\left(\mathrm{e}^{\mathrm{j} \omega} I-\mathbb{A}\right)^{-1} \mathbb{B}_{0} \\
I
\end{array}\right] \prec 0, \quad \forall \omega \in\left[\omega_{l}, \omega_{u}\right]
$$

ii) There exist $\mathcal{Q} \succ 0$ and a symmetric $\mathcal{P}$ such that

$$
\left[\begin{array}{cc}
\mathbb{A} & \mathbb{B}_{0} \\
I & 0
\end{array}\right]^{\top}(\Phi \otimes \mathcal{P}+\Psi \otimes \mathcal{Q})\left[\begin{array}{cc}
\mathbb{A} & \mathbb{B}_{0} \\
I & 0
\end{array}\right]+\Theta \prec 0
$$

Lemma 2. (Gahinet and Apkarian, 1994) Given a symmetric matrix $\Gamma \in \mathbb{R}^{p \times p}$ and two matrices $\Lambda, \Sigma$ of column dimension $p$, there exists a matrix $\mathcal{W}$ such that the following inequality holds

$$
\Gamma+\operatorname{sym}\left\{\Lambda^{\top} \mathcal{W} \Sigma\right\} \prec 0,
$$

if, and only if the following two projection inequalities with respect to $\mathcal{W}$ are satisfied

$$
\Lambda^{\perp^{\top}} \Gamma \Lambda^{\perp} \prec 0, \Sigma^{\perp^{\top}} \Gamma \Sigma^{\perp} \prec 0,
$$

where $\Lambda^{\perp}$ and $\Sigma^{\perp}$ are arbitrary matrices whose columns form a basis of the null spaces of $\Lambda$ and $\Sigma$, respectively. 


\section{Background and problem formulation}

The notation for variables in this paper is of the form $w_{k}(p), 0 \leq p \leq \alpha<\infty, k \geq 0$, where $w$ is the scalar or vector-valued variable under consideration, $\alpha$ is the finite trial length and $k$ is the trial number. The plant dynamics is assumed to be discrete linear time-invariant and described in the ILC setting as

$$
\begin{aligned}
x_{k}(p+1) & =A x_{k}(p)+B u_{k}(p), \\
y_{k}(p) & =C x_{k}(p),
\end{aligned}
$$

where $x_{k}(p) \in \mathbb{R}^{n}$ is the state vector, $y_{k}(p) \in \mathbb{R}^{m}$ is the output vector and $u_{k}(p) \in \mathbb{R}^{l}$ is the control input vector. Also we define $z$ as the standard forward shift operator along discrete-time axis, i.e.,

$$
z x_{k}(p)=x_{k}(p+1) .
$$

see, e.g., the cited papers (Bristow et al., 2006) for the details of how the $z$-transform can be applied over the finite trial length without errors arising from the basic definition of this transform over an infinite interval. Hence (6) can, under the assumption that the pair $\{A, B\}$ is controllable and the pair $\{C, A\}$ observable, be equivalently represented by the transfer-function matrix

$$
G(z)=C(z I-A)^{-1} B .
$$

This paper considers system models where the input has no direct affect on the output and hence they have relative degree $r \geq 1$, i.e.,

$$
C A^{r-1} B \neq 0 \text {, and } C A^{i} B=0, \forall i=1,2, \ldots, r-2 .
$$

The form of ILC considered in this paper is shown in the block diagram of Figure 1 and consists of a unity negative feedback control loop with controller $C$ applied on the current trial $k$ and the ILC law as shown within the shaded part of this figure. In this paper no loss of generality arises

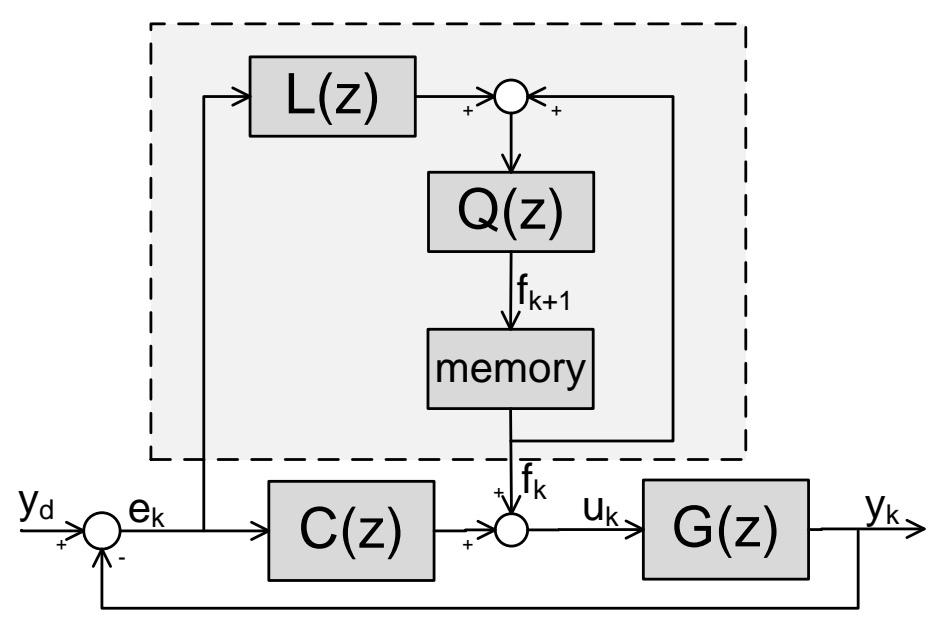

Figure 1: ILC block diagram representation.

from assuming that the initial state vector on each trial is zero. The memory block in Figure 1 represents the use of previous trial information in the computation of the current trial control input and $y_{d}$ denotes the supplied reference. In the literature $L$ is often termed the learning filter and $Q$ 
the robustness filter. All computations within the shaded part of Figure 1 are completed in the time elapsed between the end of one trial and the beginning of the next, i.e., off-line.

The first task is to find guidelines for the choice of $C, L$ and $Q$, where to improve learning and robustness a basic rule is to choose $Q$ as a low-pass filter whose magnitude is unity for the frequency range where reference tracking is required and zero at all other frequencies. From the block diagram of Figure 1, the ILC law is

$$
F_{k+1}(z)=Q(z)\left(F_{k}(z)+L(z) E_{k}(z)\right)
$$

and

$$
E_{k}(z)=(I+G(z) C(z))^{-1} Y_{d}(z)-(I+G(z) C(z))^{-1} G(z) F_{k}(z) .
$$

Hence, the previous trial error feedforward contribution (assuming $Y_{d}(z)=0$ ) to the current trial error is

$$
E_{k}(z)=-\left[(I+G(z) C(z))^{-1} G(z)\right] F(z)=-S_{P}(z) F_{k}(z),
$$

where $S_{P}(z)=(I+G(z) C(z))^{-1} G(z)$ denotes the sensitivity function and the propagation of the error from trial-to-trial is

$$
E_{k+1}(z)=Q(z)\left(I-S_{P}(z) L(z)\right) E_{k}(z)
$$

Suppose now that trial-to-trial error convergence holds, where the condition for this property is developed below, and let $E_{\infty}=\lim _{k \rightarrow \infty} E_{k}$. Then

$$
E_{\infty}(z)=((I-Q(z))(I+G(z) C(z))+G(z) Q(z) L(z))^{-1}(I-Q(z)) Y_{d}(z),
$$

and for ease of notation introduce

$$
M(z)=Q(z)\left(I-S_{P}(z) L(z)\right)
$$

and hence

$$
E_{k+1}(z)-E_{k}(z)=M(z)\left(E_{k}(z)-E_{k-1}(z)\right)
$$

or

$$
E_{k+1}(z)-E_{\infty}(z)=M(z)\left(E_{k}(z)-E_{\infty}(z)\right) .
$$

Let $\|\cdot\|_{\infty}$ denote the $\mathcal{H}_{\infty}$ norm and let $\bar{\sigma}(\cdot)$ denote the maximum singular value of its matrix argument. Then trial-to-trial error convergence has been formulated in $\mathcal{H}_{\infty}$ control terms (see also, for example, (Bristow et al., 2006; Doh and Ryoo, 2008)). Hence the trial-to-trial error converges monotonically in $k$ provided

$$
\|M(z)\|_{\infty} \triangleq \sup _{\omega \in[-\pi, \pi]} \bar{\sigma}\left(M\left(\mathrm{e}^{\mathrm{j} \omega}\right)\right)<1,
$$

and minimizing $\|M(z)\|_{\infty}$ increases the convergence speed.

Remark 1. Using (9) perfect tracking, i.e., $e_{\infty}(p)=0$ over the trial length is only achieved when $Q(z)=I$. This choice is, however, prone to the effects of high frequency noise and non-repeating disturbances. Hence, $Q$ has to be chosen as a low-pass filter with (ideally) unity magnitude for low frequencies and zero magnitude for high frequencies, i.e., perfect tracking of the reference is achieved in the specified frequency range and attenuated over the remainder. Specifically, when considering high frequencies (i.e., when $Q(z)=0$ ), (9) reduces to

$$
E_{\infty}(z)=(I+G(z) C(z))^{-1} Y_{d}(z) .
$$

This means that $Q$-filter cut-off frequency should be larger than (or at least equal to) the desired close-loop bandwidth. Note that in some cases, $Q$ can be taken as a zero-phase filter and therefore enables high-frequency attenuation without introducing any phase-lag, see, e.g., (Bristow et al., 2006; de Roover, 1996) for a detailed treatment of this choice. 
The structure of (10) gives that fast monotonic convergence will result if $L(z)=S_{p}^{-1}(z)$. Also the feedback controller $C$ must ensure that $(I+G(z) C(z))^{-1} \approx 0$. In applications, however, the choice of $C(z), L(z)$ and $Q(z)$ is somewhat more complex and this problem is considered again later in this paper.

\subsection{ILC as a repetitive process}

Having obtained the requirements for ILC design, a number of settings can be used to complete the design. Previous work has shown that using repetitive process setting has advantages when specifications beyond trial-to-trial error convergence are present. This section formulates the ILC design problem considered in the repetitive process setting, starting with the dynamics of these processes.

The state-space model of a discrete linear repetitive process (Rogers et al., 2007) over the pass length $\alpha$ is

$$
\begin{aligned}
x_{k+1}(p+1) & =\mathbb{A} x_{k+1}(p)+\mathbb{B} u_{k+1}(p)+\mathbb{B}_{0} y_{k}(p), \\
y_{k+1}(p) & =\mathbb{C} x_{k+1}(p)+\mathbb{D} u_{k+1}(p)+\mathbb{D}_{0} y_{k}(p),
\end{aligned}
$$

where on pass $k$ where $x_{k}(p) \in \mathbb{R}^{n}$ is the state vector, $y_{k}(p) \in \mathbb{R}^{m}$ is the output vector and $u_{k}(p) \in \mathbb{R}^{l}$ is the control input vector. To complete the process description it is necessary to specify the boundary conditions, i.e., the pass state initial vector sequence $x_{k}(0), k \geq 1$, and the initial pass profile $y_{0}(p)$. In this paper, the initial state vector on each pass is taken as the zero vector and the initial pass profile vector entries are assumed to be specified over the pass length as known functions of $p$.

The terms $\mathbb{B}_{0} y_{k}(p)$ and $\mathbb{D}_{0} y_{k}(p)$ in (11) represent the contributions of the previous pass profile vector to the current pass state and pass profile vectors respectively. This inter-pass interaction is the source of the unique control problem for these processes where the sequence of pass profiles $\left\{y_{k}\right\}_{k \geq 1}$ can contain oscillations that increase in amplitude from pass-to-pass, i.e., with increasing $k$. The stability theory (Rogers et al., 2007) for these processes requires that a bounded initial pass profile produces a bounded sequence of pass profiles, where in the strongest form this property is required for all possible values of the pass length. In the remainder of this paper trial is replaced by pass to conform with ILC terminology.

To formulate the ILC design problem in the repetitive process terms assume $Q=I$ (at this stage frequency attenuation using the $Q$-filer is not considered) and rewrite (10) as

$$
M(z)=(I+G(z) C(z))^{-1}[G(z) C(z) G(z) L(z)+(I+G(z) C(z))(I-G(z) L(z))],
$$

or

$$
M(z)=I-G(z)\left[\begin{array}{ll}
C(z) & L(z)
\end{array}\right]\left(\left[\begin{array}{cc}
(I+G(z) C(z)) & G(z) L(z) \\
0 & I
\end{array}\right]\right)^{-1}\left[\begin{array}{l}
0 \\
I
\end{array}\right] .
$$

Moreover, $M(z)$ can be represented as a particular case of a general control configuration (Skogestad and Postlethwaite, 2005), where the generalized plant $P$, i.e., the interconnection system of the controlled system, is

$$
P(z)=\left[\begin{array}{c|c}
P_{11}(z) & P_{12}(z) \\
\hline P_{21}(z) & P_{22}(z)
\end{array}\right]=\left[\begin{array}{c|c}
I & -G(z) \\
\hline 0 & -G(z) \\
I & 0
\end{array}\right],
$$

which is the transfer-function matrix from $\left[e_{k}^{\top} u_{k+1}^{\top}\right]^{\top}$ to $\left[e_{k+1}^{\top} y_{k+1}^{\top}\right]^{\top}$, where $y_{k+1}=\left[\left(\bar{y}_{k+1}\right)^{\top}\left(\widehat{y}_{k+1}\right)^{\top}\right]^{\top}$. The generalized ILC controller to be found is given by $K(z)=\left[\begin{array}{lll}C(z) & L(z)\end{array}\right]$ and hence $M(z)$ can be rewritten as

$$
M(z)=P_{11}(z)+P_{12}(z) K(z)\left(I-P_{22}(z) K(z)\right)^{-1} P_{21}(z) .
$$


This last formula shows that the transfer-function matrix from $e_{k}$ to $e_{k+1}$ is given by a linear fractional transformation (LFT) of $P(z)$ with $K(z)$ as the parameter. Furthermore, the state-space representation of (12) is

$$
\begin{aligned}
x_{k+1}(p+1) & =A x_{k+1}(p)+B u_{k+1}(p), \\
\bar{y}_{k+1}(p) & =-C x_{k+1}(p), \\
\widehat{y}_{k+1}(p) & =e_{k}(p), \\
e_{k+1}(p) & =-C x_{k+1}(p)+e_{k}(p) .
\end{aligned}
$$

Also, suppose that the ILC controller $K$ is represented by the state-space model

$$
\begin{aligned}
\widetilde{x}_{k+1}(p+1) & =A_{K} \widetilde{x}_{k+1}(p)+\left[\begin{array}{ll}
B_{K 1} & B_{K 2}
\end{array}\right]\left[\begin{array}{l}
\bar{y}_{k+1}(p) \\
\widehat{y}_{k+1}(p)
\end{array}\right], \\
u_{k+1}(p) & =C_{K} \widetilde{x}_{k+1}(p)+\left[\begin{array}{ll}
D_{K 1} & D_{K 2}
\end{array}\right]\left[\begin{array}{l}
\bar{y}_{k+1}(p) \\
\widehat{y}_{k+1}(p)
\end{array}\right],
\end{aligned}
$$

which results in the controller realization $K(z)=D_{K}+C_{K}\left(z I-A_{K}\right)^{-1} B_{K}$ where

$$
B_{K}=\left[\begin{array}{ll}
B_{K 1} & B_{K 2}
\end{array}\right], D_{K}=\left[\begin{array}{ll}
D_{K 1} & D_{K 2}
\end{array}\right] .
$$

The ILC dynamics can now be written as

$$
\left[\begin{array}{c}
x_{k+1}(p+1) \\
\widetilde{x}_{k+1}(p+1) \\
\hline e_{k+1}(p)
\end{array}\right]=\left[\begin{array}{cc|c}
A-B D_{K 1} C & B C_{K} & B D_{K 2} \\
-B_{K 1} C & A_{K} & B_{K 2} \\
\hline-C & 0 & I
\end{array}\right]\left[\begin{array}{c}
x_{k+1}(p) \\
\widetilde{x}_{k+1}(p) \\
\hline e_{k}(p)
\end{array}\right],
$$

which is a discrete linear repetitive process state-space model of the form (11) with current trial state vector $\left[x_{k+1}^{\top} \widetilde{x}_{k+1}^{\top}\right]$ zero input vector and previous pass profile $e_{k}$. Treated as a repetitive process, the stability along the pass property will force the trial-to-trial error to converge monotonically to zero, see the next section.

If an example described by (6) has relative degree $r>0$, an $r$-step delay between its input and output results. To overcome this problem, the previous trial error is shifted by $r$ samples to form an the anticipative feedforward control law (Wang, 2000). In implementation, this structure means that the input signal modification $f_{k}(p)$ at time instant $p$ is paired with the error signal $e_{k}(p+r)$ at time instant $p+r$. Obviously, this is possible since the error signal from trial $k$ is available once this trial is complete. As a consequence this anticipative control law the transfer-function matrix of the learning controller is taken as $z^{r} L$ instead of $L$.

The generalized plant $P$ resulting from the above modification is

$$
P(z)=\left[\begin{array}{c|c}
P_{11}(z) & P_{12}(z) \\
\hline P_{21}(z) & P_{22}(z)
\end{array}\right]=\left[\begin{array}{c|c}
I & -G(z) \\
\hline 0 & -z^{r} G(z) \\
I & 0
\end{array}\right],
$$

where the block $P_{22}$ includes the anticipatory operator and the forward time shift is applied to the error signal transmitted through $\widehat{y}_{k+1}$ - see the 3rd equation of (13). Then, on introducing the following notation for (15),

$$
\bar{A}=\left[\begin{array}{cc}
A-B D_{K 1} C & B C_{K} \\
-B_{K 1} C & A_{K}
\end{array}\right], \bar{B}_{0}=\left[\begin{array}{c}
B D_{K 2} \\
B_{K 2}
\end{array}\right], \bar{C}=\left[\begin{array}{ll}
-C & 0
\end{array}\right], \bar{D}_{0}=I,
$$

the transfer-function matrix $M(z)$ from $e_{k}$ to $e_{k+1}$ is

$$
M(z)=\bar{C}(z I-\bar{A})^{-1} \bar{B}_{0} z^{r}+\bar{D}_{0}=\bar{C} z^{r}(z I-\bar{A})^{-1} \bar{B}_{0}+\bar{D}_{0} .
$$


Consider the term $z^{r}(z I-\bar{A})^{-1}$ and assuming $(z I-\bar{A})^{-1}$ is nonsingular $(z I-\bar{A})(z I-\bar{A})^{-1}=I$, and hence

$$
z(z I-\bar{A})^{-1}=I+\bar{A}(z I-\bar{A})^{-1} .
$$

Also

$$
z^{2}(z I-\bar{A})^{-1}=z\left(I+\bar{A}(z I-\bar{A})^{-1}\right)=z I+\bar{A}+\bar{A}^{2}(z I-\bar{A})^{-1}
$$

and therefore

$$
z^{r}(z I-\bar{A})^{-1}=\sum_{j=0}^{r-1} z^{r-1-j} \bar{A}^{j}+\bar{A}^{r}(z I-\bar{A})^{-1}
$$

Rewriting $\bar{A}$ in (16) as

$$
\bar{A}=\mathcal{A}+\mathcal{B K}=\left[\begin{array}{cc}
A & 0 \\
0 & 0
\end{array}\right]+\left[\begin{array}{cc}
B & 0 \\
0 & I
\end{array}\right]\left[\begin{array}{lll}
-D_{K 1} C & C_{K} \\
-B_{K 1} C & A_{K}
\end{array}\right]
$$

gives, on using (18),

$$
\bar{C} z^{r}(z I-\bar{A})^{-1} \bar{B}_{0}+\bar{D}_{0}=\bar{C}\left(\sum_{j=0}^{r-1} z^{r-1-j}(\mathcal{A}+\mathcal{B K})^{j}+(\mathcal{A}+\mathcal{B K})^{r}(z I-(\mathcal{A}+\mathcal{B K}))^{-1}\right) \bar{B}_{0}+\bar{D}_{0} .
$$

Also for all $r \geq 0$

$$
\bar{C} \mathcal{A}^{r} \mathcal{B}=\left[-C A^{r} B 0\right]
$$

and hence by (8)

$$
\begin{aligned}
\bar{C}(\mathcal{A}+\mathcal{B K})^{r-1} & =\bar{C} \mathcal{A}^{r-1} \\
\bar{C}(\mathcal{A}+\mathcal{B K})^{r} & =\bar{C} \mathcal{A}^{r-1}(\mathcal{A}+\mathcal{B} \mathcal{K}),
\end{aligned}
$$

or

$$
\begin{aligned}
M(z) & =\bar{C}\left(\sum_{j=0}^{r-1} z^{r-1-j}(\mathcal{A}+\mathcal{B K})^{j}+(\mathcal{A}+\mathcal{B K})^{r}(z I-(\mathcal{A}+\mathcal{B} \mathcal{K}))^{-1}\right) \bar{B}_{0}+\bar{D}_{0} \\
& =\bar{C}(\mathcal{A}+\mathcal{B K})^{r-1} \bar{B}_{0}+\bar{C}(\mathcal{A}+\mathcal{B K})^{r}(z I-(\mathcal{A}+\mathcal{B K}))^{-1} \bar{B}_{0}+\bar{D}_{0} \\
& =\bar{C} \mathcal{A}^{r-1} \bar{B}_{0}+\bar{C} \mathcal{A}^{r-1}(\mathcal{A}+\mathcal{B K})(z I-(\mathcal{A}+\mathcal{B} \mathcal{K}))^{-1} \bar{B}_{0}+\bar{D}_{0} .
\end{aligned}
$$

Hence, $M(z)$ of (17) can be rewritten as

$$
M(z)=\mathbb{C}(z I-\mathbb{A})^{-1} \mathbb{B}_{0}+\mathbb{D}_{0},
$$

where the matrices in the state-space quadruple $\left\{\mathbb{A}, \mathbb{B}_{0}, \mathbb{C}, \mathbb{D}_{0}\right\}$ are

$$
\begin{aligned}
& \mathbb{A}=(\mathcal{A}+\mathcal{B K})=\left[\begin{array}{cc}
A-B D_{K 1} C & B C_{K} \\
-B_{K 1} C & A_{K}
\end{array}\right], \mathbb{B}_{0}=\left[\begin{array}{c}
B D_{K 2} \\
B_{K 2}
\end{array}\right], \mathbb{D}_{0}=\bar{D}_{0}+\bar{C} \mathcal{A}^{r-1} \bar{B}_{0}=I-C A^{r-1} B D_{K 2}, \\
& \mathbb{C}=\bar{C} \mathcal{A}^{r-1}(\mathcal{A}+\mathcal{B K})=\left[-C A^{r}+C A^{r-1} B D_{K 1} C-C A^{r-1} B C_{K}\right] .
\end{aligned}
$$

These matrices define a discrete linear repetitive process state-space model of the form (11) and will be used to formulate the ILC design problem in terms of repetitive process stability theory.

The 2D/repetitive processes setting is well-established in the design and experimental validation of ILC laws but the currently available results do have limitations that, if removed, would further 
strengthen their applicability for end users. Firstly, many of the available results, e.g., Hładowski et al. (2010); Paszke et al. (2013) use state feedback control and therefore experimental implementation or a physical application requires that all entries in the current trial state vector are available for measurement, or a state estimator is used, in addition to the requirement that these measurements are not noise corrupted. Also, a static gain used as the learning filter does not enable satisfactory performance in some cases since the same gain is used for entire frequency range. Only a limited number of papers focus on imposing different design requirements in various frequency ranges, which is of considerable practical significance since performance issues can differ over different frequency ranges.

It is rarely case that a systematic procedure for designing the $Q$-filter is obtained. Increasing the order of the $Q$-filter results in a higher order feedback and learning controller and this is not desirable in many practical applications. Also it is common that the $Q$-filter has to be chosen prior to applying the ILC design procedure and therefore it is almost impossible to perfectly choose its bandwidth. Moreover, most of the available design algorithms can impose conservative stability conditions since they use a constant frequency independent Lyapunov matrix condition that must hold for the entire frequency range.

This paper continues the development of the repetitive process setting for ILC design and overcomes the problems listed above. The design makes use of a general control configuration and allows the computation of ILC controller by solving a stability problem for discrete repetitive processes over finite frequency ranges. This approach is non-trivial since the conditions of finite-frequency control impose several difficulties in the derivation. These include how to construct the state-space representation of closed-loop system when an anticipative control law is applied and the generalized ILC controller has full dynamic form and does not have the state vector available for measurement. Also, some bilinear terms are decoupled and as the result the required learning and feedback controllers are computed through the solution of convex optimization problems by LMIs. Moreover, the generalized version of the KYP lemma is used to permit ILC design over finite frequency ranges. Finally, a systematic procedure for designing the robustness filter is developed.

\section{ILC design}

In this section, the stability theory for discrete linear repetitive processes (Rogers et al., 2007) is used to develop a new ILC design algorithm. The stability theory for linear repetitive processes has been developed using an abstract model in a Banach space setting that includes all examples as special cases. For processes described by (11) the trial-to-trial coupling is the convolution operator for a discrete standard linear system with state-space model matrices $\left\{\mathbb{A}, \mathbb{B}_{0}, \mathbb{C}_{0}, \mathbb{D}_{0}\right\}$. Hence the contribution from trial $k$ to trial $k+1$ can be written as $y_{k+1}=L_{\alpha} y_{k}, k \geq 0$. Also let $y_{k} \in E_{\alpha}$, where $E_{\alpha}$ is a suitably chosen Banach space with norm denoted by $\|\cdot\|$ and let the same symbol denote the induced norm on the bounded linear operator $L_{\alpha}$.

Asymptotic stability for linear repetitive processes is equivalent to the existence of finite real scalars $M_{\alpha}>0$ and $\lambda_{\alpha} \in(0,1)$ such that $\left\|L_{\alpha}^{k}\right\| \leq M_{\alpha} \lambda_{\alpha}^{k}, k \geq 0$. For examples described by (11), asymptotic stability requires that $\rho\left(\mathbb{D}_{0}\right)<1$ and if this condition holds the sequence of trial profiles produced will converge in $k$ to a standard discrete linear system, termed the limit profile, with state matrix $\mathbb{A}_{l p}=\mathbb{A}+\mathbb{B}_{0}\left(I-\mathbb{D}_{0}\right)^{-1} \mathbb{C}$. Consider, however, the special case when $\mathbb{A}=0.5, \mathbb{B}=1$, $\mathbb{B}_{0}=$ $-0.5+\beta, \mathbb{C}=1$ and $\mathbb{D}_{0}=0$. Then $\mathbb{A}_{l p}=\beta$ and hence the limit profile is unstable in $p$, i.e., along the trial when $|\beta| \geq 1$.

The existence of examples such as the one given above is due to the finite trial length, a property of the process and not an assumption imposed for analysis purposes. Stability along the trial prevents such examples from arising by imposing the stability property for all possible values of the finite 
trial length. This property requires the existence of finite real scalars $M_{\infty}>0$ and $\lambda_{\infty} \in(0,1)$, which are independent of $\alpha$, such that $\left\|L_{\alpha}^{k}\right\| \leq M_{\infty} \lambda_{\infty}^{k}, k \geq 0$. Since the numbers $M_{\infty}$ and $\lambda_{\infty}$ are independent of $\alpha$ means that the entries in $\left\{y_{k}\right\}$ converge monotonically to zero as $k \rightarrow \infty$ and in the ILC application this enforces monotonic trial-to-trial error convergence to zero.

The following result characterizes stability along the pass of examples described by (11).

Lemma 3. (Rogers et al., 2007) A discrete linear repetitive process described by (11) and (19) is stable along the trial if and only if

i) $\rho\left(\mathbb{D}_{0}\right)<1$,

ii) $\rho(\mathbb{A})<1$,

iii) all eigenvalues of $M\left(\mathrm{e}^{\mathrm{j} \omega}\right)=\mathbb{C}\left(\mathrm{e}^{\mathrm{j} \omega} I-\mathbb{A}\right)^{-1} \mathbb{B}_{0}+\mathbb{D}_{0}, \forall \omega \in[-\pi, \pi]$ have modulus strictly less than unity.

As described above, the first condition in this last result is the necessary and sufficient condition for asymptotic stability. This condition is independent of the along the trial dynamics and holds even if the state matrix has eigenvalues on or outside the unit circle in the complex plane. Even if this matrix is required to be stable in the standard discrete linear systems sense then this does not guarantee stability along the trial. To achieve stability along the trial also requires condition iii).

The following is the first new result of this paper and its proof makes use of results in (Paszke and Bachelier, 2013; Paszke et al., 2013).

Theorem 1. An ILC scheme described as a discrete linear repetitive process of the form (11) and (19) is stable along the trial and hence monotonic trial-to-trial error convergence occurs if there exist matrices $S \succ 0, \mathcal{W}$ and a symmetric matrix $\mathcal{P}$ such that the following matrix inequalities are feasible

$$
\begin{gathered}
{\left[\begin{array}{ccc}
S-\mathcal{W}-\mathcal{W}^{\top} & \mathcal{W}^{\top} \mathbb{A} \\
\mathbb{A}^{\top} \mathcal{W} & -S
\end{array}\right] \prec 0} \\
{\left[\begin{array}{cccc}
-\mathcal{P} & -\mathcal{W} & 0 & 0 \\
-\mathcal{W}^{\top} & \mathcal{P}+\mathbb{A}^{\top} \mathcal{W}+\mathcal{W}^{\top} \mathbb{A} & \mathcal{W}^{\top} \mathbb{B}_{0} & \mathbb{C}^{\top} \\
0 & \mathbb{B}_{0}^{\top} \mathcal{W} & -I & \mathbb{D}_{0}^{\top} \\
0 & \mathbb{C} & \mathbb{D}_{0} & -I
\end{array}\right] \prec 0 .}
\end{gathered}
$$

Proof. Assume that (20) and (21) hold. Then $\mathcal{W}+\mathcal{W}^{\top} \succ S \succ 0$ which implies that $\mathcal{W}$ is non-singular and hence invertible. Next, rewrite (20) as

$$
\left[\begin{array}{cc}
S & 0 \\
0 & -S
\end{array}\right]+\operatorname{sym}\left\{\left[\begin{array}{l}
-I \\
\mathbb{A}^{\top}
\end{array}\right] \mathcal{W}\left[\begin{array}{ll}
I & 0
\end{array}\right]\right\} \prec 0
$$

and a direct application of Lemma 2 yields $\mathbb{A}^{\top} S \mathbb{A}-S \prec 0$. Hence, from the Lyapunov stability theory for standard discrete linear systems, condition ii) of Lemma 3 holds. Next, application of the Schur's complement formula enables (21) to be rewritten as

$$
\left[\begin{array}{ccc}
-\mathcal{P} & 0 & 0 \\
0 & \mathcal{P}+\mathbb{C}^{\top} \mathbb{C} & \mathbb{C}^{\top} \mathbb{D}_{0} \\
0 & \mathbb{D}_{0}^{\top} \mathbb{C} & \mathbb{D}_{0}^{\top} \mathbb{D}_{0}-I
\end{array}\right]+\operatorname{sym}\left\{\Lambda^{\top} \mathcal{W} \Sigma\right\} \prec 0
$$

where

$$
\Lambda=\left[-I \mathbb{A}_{0}\right], \Sigma=\left[\begin{array}{lll}
0 & I & 0
\end{array}\right]
$$


and $\mathcal{W}$ is a slack matrix variable. Moreover, (22) is in the form of (4) since $\Lambda^{\perp}$ and $\Sigma^{\perp}$ can be chosen as

$$
\Lambda^{\perp}=\left[\begin{array}{cc}
\mathbb{A} & \mathbb{B}_{0} \\
I & 0 \\
0 & I
\end{array}\right], \Sigma^{\perp}=\left[\begin{array}{ll}
I & 0 \\
0 & 0 \\
0 & I
\end{array}\right] .
$$

Hence, by the result of Lemma 2, (22) holds if and only if

$$
\left[\begin{array}{lll}
\mathbb{A}^{\top} & I & 0 \\
\mathbb{B}_{0}^{\top} & 0 & I
\end{array}\right]\left[\begin{array}{ccc}
-\mathcal{P} & 0 & 0 \\
0 & \mathcal{P}+\mathbb{C}^{\top} \mathbb{C} & \mathbb{C}^{\top} \mathbb{D}_{0} \\
0 & \mathbb{D}_{0}^{\top} \mathbb{C} & \mathbb{D}_{0}^{\top} \mathbb{D}_{0}-I
\end{array}\right]\left[\begin{array}{cc}
\mathbb{A} & \mathbb{B}_{0} \\
I & 0 \\
0 & I
\end{array}\right] \prec 0,
$$

and

$$
\left[\begin{array}{cc}
-\mathcal{P} & 0 \\
0 & \mathbb{D}_{0}^{\top} \mathbb{D}_{0}-I
\end{array}\right] \prec 0
$$

Moreover, for (23) and (24) to hold, $\mathbb{D}_{0}^{\top} \mathbb{D}_{0}-I \prec 0$ and hence that condition $i$ ) of Lemma 3 holds. Moreover, (23) can be written in the form (3) where

$$
\Theta=\left[\begin{array}{cc}
\mathbb{C} & \mathbb{D}_{0} \\
0 & I
\end{array}\right]^{\top}\left[\begin{array}{cc}
I & 0 \\
0 & -I
\end{array}\right]\left[\begin{array}{cc}
\mathbb{C} & \mathbb{D}_{0} \\
0 & I
\end{array}\right],
$$

and hence (2) for this case with $\Theta$ defined above is equivalent to

$$
\left[\begin{array}{c}
M\left(\mathrm{e}^{\mathrm{j} \omega}\right) \\
I
\end{array}\right]^{*}\left[\begin{array}{cc}
I & 0 \\
0 & -I
\end{array}\right]\left[\begin{array}{c}
M\left(\mathrm{e}^{\mathrm{j} \omega}\right) \\
I
\end{array}\right] \prec 0, \quad \forall \omega \in[-\pi, \pi]
$$

or

$$
M\left(\mathrm{e}^{\mathrm{j} \omega}\right)^{*} M\left(\mathrm{e}^{\mathrm{j} \omega}\right) \prec I, \quad \forall \omega \in[-\pi, \pi] .
$$

Hence condition iii) of Lemma 3 holds and the proof is complete.

Remark 2. The inequality (25) can be rewritten as

$$
M\left(\mathrm{e}^{\mathrm{j} \omega}\right)^{*} M\left(\mathrm{e}^{\mathrm{j} \omega}\right) \prec \mu^{2} I, \quad \forall \omega \in[-\pi, \pi],
$$

where $0<\mu \leq 1$. Then $\bar{\sigma}\left(M\left(\mathrm{e}^{\mathrm{j} \omega}\right)\right)<\mu$ and by minimizing $\mu$ a higher speed of monotonic trial-to-trial error convergence is obtained.

The advantage of this new result is that the product terms involving $\mathcal{P}, S$ and process state-space model matrices are decoupled by the slack matrix variable $\mathcal{W}$ and this provides an additional degree of freedom. Also this additional matrix variable is not required to be symmetric. The inequalities in Theorem 1 are not LMIs as they are bilinear in $\mathcal{W}$ and the matrices defining the controller $K$. This means no computationally effective method exists for checking (20) and (21). However, by using a particular set of transformations, the inequality (21) can be converted into an LMI.

Assume that the auxiliary slack matrix variable $\mathcal{W}$ and its inverse are partitioned into blocks as (see (Oliveira et al., 2002) for more details on the application of this step)

$$
\mathcal{W}=\left[\begin{array}{ll}
X & ? \\
U & ?
\end{array}\right], \quad \mathcal{W}^{-1}=\left[\begin{array}{ll}
N & ? \\
R & ?
\end{array}\right]
$$

where '?' is used to denote block entries in these matrices that are not involved in the derivations that follow. Also suppose, without loss of generality, that $U$ and $R$ are invertible and define the transformation matrices $F_{1}$ and $F_{2}$ as

$$
F_{1}=\left[\begin{array}{ll}
X & I \\
U & 0
\end{array}\right], F_{2}=\left[\begin{array}{cc}
I & N \\
0 & R
\end{array}\right]
$$


where $\mathcal{W} F_{2}=F_{1}$ and introduce the notation

$$
\begin{aligned}
& \widehat{W}=F_{2}^{\top} \mathcal{W} F_{2}=\left[\begin{array}{cc}
X & I \\
Z^{\top} & N^{\top}
\end{array}\right], \widehat{A}=F_{2}^{\top} \mathcal{W}^{\top} \mathbb{A} F_{2}=\left[\begin{array}{cc}
X^{\top} A-\widetilde{B}_{1} C & \widetilde{A} \\
A-B D_{K 1} C & A N+B \widetilde{C}
\end{array}\right], \\
& \widehat{B}_{0}=F_{2}^{\top} \mathcal{W}^{\top} \mathbb{B}_{0}=\left[\begin{array}{c}
\widetilde{B}_{2} \\
B D_{K 2}
\end{array}\right], \widehat{C}=\mathbb{C} F_{2}=\left[-C A^{r-1}\left(A-B D_{K 1} C\right)-C A^{r-1}(A N+B \widetilde{C})\right],
\end{aligned}
$$

where

$$
\begin{aligned}
\widetilde{A} & =X^{\top} A N-X^{\top} B D_{K 1} C N-U^{\top} B_{K 1} C N+X^{\top} B C_{K} R+U^{\top} A_{K} R, Z=X^{\top} N+U^{\top} R, \\
\widetilde{B}_{1} & =X^{\top} B D_{K 1}+U^{\top} B_{K 1}, \widetilde{B}_{2}=X^{\top} B D_{K 2}+U^{\top} B_{K 2}, \widetilde{C}=C_{K} R-D_{K 1} C N .
\end{aligned}
$$

The theorem established next reformulates the result of Theorem 1 to an LMI-based characterization for the existence of an ILC controller $K$ represented by (14).

Theorem 2. An ILC scheme described as a discrete linear repetitive process of the form (11) and (19) is stable along the trial and hence monotonic trial-to-trial error convergence occurs if there exist matrices $\widetilde{A}, \widetilde{B}_{1}, \widetilde{B}_{2}, \widetilde{C}, D_{K 1}, D_{K 2}, N, X, Z$ of the form (27), $\widehat{S} \succ 0$ and a symmetric matrix $\widehat{\mathcal{P}}$ such that the following LMIs are feasible

$$
\begin{gathered}
{\left[\begin{array}{ccc}
\widehat{S}-\widehat{W}-\widehat{W}^{\top} & \widehat{A} \\
\widehat{A}^{\top} & -\widehat{S}
\end{array}\right] \prec 0,} \\
{\left[\begin{array}{cccc}
-\widehat{\mathcal{P}} & -\widehat{W} & 0 & 0 \\
-\widehat{W}^{\top} & \widehat{\mathcal{P}}+\widehat{A}^{\top}+\widehat{A} & \widehat{B}_{0} & \widehat{C}^{\top} \\
0 & \widehat{B}_{0}^{\top} & -I & \mathbb{D}_{0}^{\top} \\
0 & \widehat{C} & \mathbb{D}_{0} & -I
\end{array}\right] \prec 0 .}
\end{gathered}
$$

Proof. Suppose that LMIs (28) and (29) are feasible. Then $\widehat{W}+\widehat{W}^{\top} \succ \widehat{S} \succ 0$ which implies that $\widehat{W}$ defined by (27) is non-singular and hence invertible. Next post- and pre-multiply (28) by $\operatorname{diag}\left\{F_{2}^{-1}, F_{2}^{-1}\right\}$ to obtain (20). Moreover, the LMI (29) results from left and right multiplying (21) by $\operatorname{diag}\left\{F_{2}, F_{2}, I, I\right\}$ and its transpose, respectively. Setting $\widehat{\mathcal{P}}=F_{2}^{\top} \mathcal{P} F_{2}, \widehat{S}=F_{2}^{\top} S F_{2}$ completes the proof.

Suppose that the LMIs (28) and (29) are feasible. Then the following is a systematic procedure for obtaining the corresponding controller matrices of (14).

1. Compute the singular value decomposition (SVD) of $Z-X^{\top} N$ to obtain square and invertible matrices $U_{1}, V_{1}$ such that $Z-X^{\top} N=U_{1} \Sigma_{1} V_{1}^{\top}$.

2. Choose the matrices $U$ and $R$ as $U^{\top}=U_{1} \Sigma_{1}^{\frac{1}{2}}, R=\Sigma_{1}^{\frac{1}{2}} V_{1}^{\top}$.

3. Compute the matrices of the ILC controller state-space model (14) using

$$
\begin{aligned}
C_{K} & =\left(\widetilde{C}+D_{K 1} C N\right) R^{-1}, \\
B_{K 1} & =U^{-\top}\left(\widetilde{B}_{1}-X^{\top} B D_{K 1}\right), \\
B_{K 2} & =U^{-\top}\left(\widetilde{B}_{2}-X^{\top} B D_{K 2}\right), \\
A_{K} & =U^{-\top}\left(\widetilde{A}-X^{\top} A N+X^{\top} B D_{K 1} C N+U^{\top} B_{K 1} C N-X^{\top} B C_{K} R\right) R^{-1},
\end{aligned}
$$

where $U^{-\top}=\left(U^{-1}\right)^{\top}=\left(U^{\top}\right)^{-1}$. 


\subsection{ILC with design specifications over finite frequency ranges}

In applications terms a critical problem is to achieve the desired shape of the maximum singular value of $M\left(\mathrm{e}^{\mathrm{j} \omega}\right)$ over the complete range to account for the spectra of exogenous signals, to penalize regulated variables and to specify the amount of plant model uncertainty, see, e.g., (Inaba et al., 2009; Tayebi et al., 2008) for further discussion on this requirement in the standard and ILC settings respectively. A common approach to ensuring that the resulting frequency specifications are satisfied is to include weighting filters in the general plant (12). The choice and tuning of these weighting filters is not trivial task and their inclusion increases the order of the general plant and hence the ILC controller, thus increasing the complexity of the design.

The objective now considered is to develop an approach for shaping the maximum singular value of $M\left(\mathrm{e}^{\mathrm{j} \omega}\right)$ to meet the control specifications in different frequency ranges without introducing weighting filters, where instead of considering the entire frequency range $[-\pi, \pi]$ attention can be limited to $\omega \in[0, \pi]$ only. Moreover, divide this frequency range into $H$ intervals (not necessarily containing the same number of frequencies) such that

$$
[0, \pi]=\bigcup_{h=1}^{H}\left[\omega_{h-1}, \omega_{h}\right],
$$

where $\omega_{0}=0$ and $\omega_{H}=\pi$. Then Lemma 1 can be applied at any frequency in any of these intervals.

The possibility to specify different performance specifications has considerable practical significance since common performance issues occur over different frequency ranges. For example, trial-totrial error convergence rate is in the 'low' frequency range whereas low sensitivity to disturbances and sensor noise are in 'high' frequency range. These objectives are included in the requirement that

$$
\bar{\sigma}\left(M\left(\mathrm{e}^{\mathrm{j} \omega}\right)\right)<\mu_{h}, \forall \omega \in\left[\omega_{h-1}, \omega_{h}\right], h=1, \ldots, H,
$$

where entire frequency range is partitioned according to (31) and

$$
0<\mu_{h} \leq 1, h=1, \ldots, H .
$$

The choice of the frequency intervals and the $\mu_{h}$ are determined by knowledge of the application under consideration.

Making use of Lemma 1 gives the following result for design to meet the specifications of (32).

Theorem 3. Consider an ILC scheme described as a discrete linear repetitive process of the form (11) and (19). Furthermore, suppose that the entire frequency range is arbitrarily divided into $H$ possible different frequency intervals as in (31). Then i) the resulting repetitive process is stable along the trial, ii) monotonic trial-to-trial error convergence occurs and iii) the finite frequency performance specifications (32) are satisfied if there exist matrices $\widetilde{A}, \widetilde{B}_{1}, \widetilde{B}_{2}, \widetilde{C}, D_{K 1}, D_{K 2}, N, X, Z$ of the form (27), $\widehat{\mathcal{Q}}_{h} \succ 0, \widehat{S} \succ 0$, symmetric $\widehat{\mathcal{P}}_{h}$ and arbitrary chosen scalars $\mu_{h}$ satisfying (33) such that the following LMIs are feasible

$$
\begin{gathered}
{\left[\begin{array}{cc}
\widehat{S}-\widehat{W}-\widehat{W}^{\top} & \widehat{A} \\
\widehat{A}^{\top} & -\widehat{S}
\end{array}\right] \prec 0,} \\
{\left[\begin{array}{cccc}
-\widehat{\mathcal{P}}_{h}-\widehat{W}-\widehat{W}^{\top} & \mathrm{e}^{\mathrm{j} \omega_{c h}} \widehat{\mathcal{Q}}_{h}-\widehat{W} & 0 & 0 \\
\mathrm{e}^{-\mathrm{j} \omega_{c h}} \widehat{\mathcal{Q}}_{h}-\widehat{W}^{\top} & \widehat{\mathcal{P}}_{h}-2 \cos \left(\omega_{d h}\right) \widehat{\mathcal{Q}}_{h}+\widehat{A}^{\top}+\widehat{A} & \widehat{B}_{0} & \widehat{C}^{\top} \\
0 & \widehat{B}_{0}^{\top} & -\mu_{h}^{2} I & \mathbb{D}_{0}^{\top} \\
0 & \widehat{C} & \mathbb{D}_{0} & -I
\end{array}\right] \prec 0,}
\end{gathered}
$$


for all $h=1, \ldots, H$, where

$$
\omega_{c h}=\frac{\omega_{h-1}+\omega_{h}}{2}, \omega_{d h}=\frac{\omega_{h}-\omega_{h-1}}{2} .
$$

Moreover, if these LMIs are feasible, the ILC controller (14) can be obtained from (30).

Proof. This follows that for Theorem 2 where $\Psi$ is chosen as in (1) for each of $H$ frequency ranges satisfying (31) with frequency specifications given in (32). Since $\bar{\sigma}\left(M\left(\mathrm{e}^{\mathrm{j} \omega}\right)\right)<1$ in each frequency interval, it follows from $(31)$ that $\bar{\sigma}\left(M\left(\mathrm{e}^{\mathrm{j} \omega}\right)\right)<1$ holds over entire frequency range the proof is complete.

Remark 3. Based on results in (Iwasaki and Hara, 2005), the decision variables $\widehat{\mathcal{P}}_{h}$ and $\widehat{\mathcal{Q}}_{h}$ have to be Hermitian matrices. However, more recent developments in (Pipeleers et al., 2011) show that $\widehat{\mathcal{P}}_{h}$ and $\widehat{\mathcal{Q}}_{h}$ can be selected as real symmetric matrices without additional conservatism, which reduces the computational load.

Remark 4. The matrix variable $\widehat{W}$ is common to all the LMI constraints in (34) and this may introduce a level of conservatism into the design. If this is the case, one possible option is to use piecewise constant matrix variables $\widehat{\mathcal{Q}}_{h} \succ 0$ and $\widehat{\mathcal{P}}_{h} \forall h=1, \ldots, H$, over the a priori chosen frequency ranges.

\section{2. $Q$-filter design procedure}

For ease of presentation, the discussion in this section deals with the single-input single-output case (when the maximum singular value can be replaced by the modulus). In this case, it follows from (10) that for low frequencies it is (relatively) easy to obtain $\left|M\left(e^{\mathrm{j} \omega}\right)\right|<1$ since in this frequency range the sensitivity function satisfies $\left|S_{P}(z)\right| \approx 0$. When the feedback becomes less effective and $\left|S_{P}\right| \approx 1$, the learning filter $L$ has to be chosen as the inverse of $S_{P}$ and if this transfer-function is strictly proper its exact inverse is improper.

The plant $G$ of (6) has a strictly proper transfer-function and therefore its exact inverse is improper. Hence it is only possible to ensure that $|Q(z)| \approx 1$ over the frequency range where $L$ is a good approximation to $G^{-1}$. Also from Theorem 3 it is possible to determine the frequency range where $\bar{\sigma}\left(M\left(\mathrm{e}^{\mathrm{j} \omega}\right)\right)<1$ and perform learning over this frequency range only. The remaining frequencies should be cut-off by the $Q$-filter because the inverse of the plant $G$ is not sufficiently matched at higher frequencies. Therefore, the $Q$-filter can be chosen as a low-pass filter (which can be implemented as the zero-phase filter, e.g., by using the filtfilt routine in MATLAB) with cut-off frequency equal to $\omega_{H}$, i.e., the highest frequency for which the result of Theorem 3 is valid.

\subsection{Design Computation}

In terms of solvability, the LMI condition of Theorem 2 (and then extended to finite frequency case in Theorem 3) is a version of the solution to the full order (the controller has the same order as the generalized plant) dynamic output-feedback control problem first reported in the non ILC literature by de Oliveira et al. (2002). As in this previous work, the result of Theorem 2 (and that of Theorem 3) is obtained at the expense of imposing some constraints on the extra matrix variable, e.g., the variable $Z$ is defined as $Z=X^{T} N+U^{T} R$ and hence some of the matrix variables in this equation are not free. Although this still imposes some degree of conservatism on the results, the number of free parameters in the new design procedure developed is significantly increased in comparison with the available techniques for ILC design in the repetitive process setting (see also the detailed explanation given in de Oliveira et al. (2002) for the non ILC case). This means that the level of conservatism is lower when compared to alternatives. 
The solution strongly depends on the design specifications imposed on the system. As highlighted in Section 3.2 on $Q$-filter design, the solution is found in relatively easy way when the performance specifications only lie in the low frequency range since the process sensitivity function satisfies $S_{P}(z) \approx$ 0 . When the feedback becomes less effective and $S_{P}(z) \approx 1$, the learning filter $L$ has to be chosen as the inverse of $S_{P}$ and if this transfer-function is strictly proper its exact inverse is improper. This last fact means that it is hard to find a solution in high frequency range. Therefore the $Q$ filter has to be used to cut-off these frequency ranges for which performance specification cannot be satisfied. Conversely, to achieve greater reduction of the tracking error, $S_{P}(z)$ needs to be small at low frequency. However, this can be done only at the cost of increasing of $S_{P}(z)$ in a different frequency region and hence the system's closed-loop bandwidth is reduced. This is a direct consequence of the analytic design tradeoff associated with the feedback loop, known as the Bode waterbed effect, see, e.g., Skogestad and Postlethwaite (2005) for more details in the non ILC case. In particular, there is always a fundamental trade off and limitation between bandwidth, robustness and tracking error.

\section{Experimental Case Study}

To validate the new design procedure, the results of an experimental validation on a laboratory servomechanism system are given and discussed. The system consists of a DC motor and the inertial mass (brass cylinder, weight $2.03[\mathrm{~kg}]$, diameter $0.066[\mathrm{~m}]$, length $0.068[\mathrm{~m}]$ ), which are connected through a rigid shaft. Rotational motion of the mass is exited by the DC motor, load position is measured by an incremental encoder and the whole system operates with a PC-based digital controller. A brake-out box contains an interface module that amplifies the control signals, which are transmitted from the PC to the DC motor. The hardware setup includes a data acquisition board, a command transmission unit and a computer that implements the control algorithms. The system is fully integrated with MATLAB/SIMULINK and operates in real-time and Table 1 gives its basic parameters. Commonly, the angular position of the mass is considered as the output and the

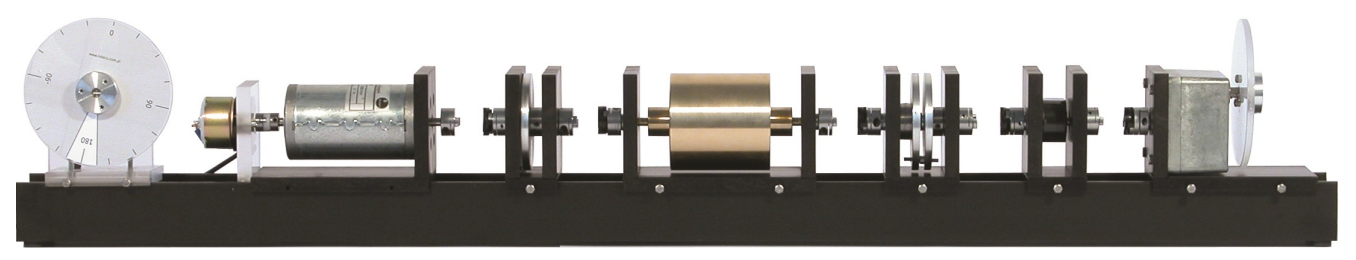

Figure 2: The Modular Servo System.

Table 1: The DC motor parameters.

\begin{tabular}{|c|c|}
\hline Rated Voltage & $24[\mathrm{~V}]$ \\
\hline Rated Output Power & $47[\mathrm{~W}]$ \\
\hline Rated Torque & $15[\mathrm{~N} \cdot \mathrm{cm}]$ \\
\hline No Load Speed & $3900\left[\mathrm{rpm} / \mathrm{min}^{-1}\right]$ \\
\hline Rated Current & $3.1[\mathrm{~A}]$ \\
\hline
\end{tabular}

armature voltage is considered as the input and hence the plant transfer-function

$$
G(s)=\frac{\Theta(s)}{V(s)}=\frac{K}{s\left((J s+b)(L s+R)+K^{2}\right)},
$$

where $K$ represents both the motor torque constant $\left(K_{t}\right)$ and the back emf constant $\left(K_{e}\right), J$ is the total moment of inertia of the rotor $\left(J_{r}\right)$ and the mass $\left(J_{m}\right), b$ is the motor viscous friction constant, 
$L$ is electric inductance and $R$ is the electric resistance. Moreover, the armature voltage of the DC motor is controlled by a PWM (pulse-width modulation) signal. As a result, the dimensionless control signal $u(t)$ is the scaled input voltage and the admissible controls satisfy $|u(t)| \leq 1$. Consequently the gain of $G(s)$ in (35) has to be increased by a factor equal to the maximum armature voltage $v_{\max }=24[\mathrm{~V}]$.

Table 2 shows the physical parameters of the DC motor and they are used to obtain the mathematical model. The transfer-function of (35) has been discretized with a sampling time of $T_{s}=0.01$

Table 2: Physical parameters of the DC motor

\begin{tabular}{|c|c|c|}
\hline$J_{r}$ & moment of inertia of the rotor & $18 \cdot 10^{-6}\left[\mathrm{~kg} \cdot \mathrm{m}^{2}\right]$ \\
\hline$J_{m}$ & moment of inertia of the mass & $0.0011\left[\mathrm{~kg} \cdot \mathrm{m}^{2}\right]$ \\
\hline $\mathrm{b}$ & motor viscous friction constant & $3.5077 \cdot 10^{-6}[\mathrm{~N} \cdot \mathrm{m} \cdot \mathrm{s}]$ \\
\hline$K_{e}$ & electromotive force constant & $0.056[\mathrm{~V} / \mathrm{rad} / \mathrm{sec}]$ \\
\hline$K_{t}$ & motor torque constant & $0.056[\mathrm{~N} \cdot \mathrm{m} / \mathrm{A}]$ \\
\hline $\mathrm{R}$ & electric resistance & $2[\Omega]$ \\
\hline $\mathrm{L}$ & electric inductance & $0.001[\mathrm{H}]$ \\
\hline
\end{tabular}

secs to give a minimal discrete linear state-space model of the form (6) with

$$
A=\left[\begin{array}{ccc}
1.0 & 0 & 0 \\
0 & 0.9860 & 0.0002 \\
0 & -0.0002 & -2.481 \cdot 10^{-8}
\end{array}\right], \quad B=\left[\begin{array}{c}
50.6240 \\
2.0613 \\
0.0119
\end{array}\right], C=\left[\begin{array}{lll}
0.0845 & -2.0613 & 0.0119
\end{array}\right] .
$$

The state matrix $A$ has two eigenvalues inside the unit circle and one on the unit circle. Hence if lifting ILC design is to be used then a stabilizing feedback control loop must first be designed. In the repetitive process setting a stabilizing control loop is part of the overall design.

The reference trajectory for the mass position is of duration 6.5 secs as shown in Figure $3(\mathrm{a})$ and its frequency spectrum is given in Figure $3(\mathrm{~b})$ and consists of harmonics from 0 to $2 \mathrm{~Hz}$. The reference signal consists of 6 revolutions in the positive direction, a return path, 6 revolutions in the negative direction and then a return to the start position. Hence the cut-off frequency of $Q$-filter must be
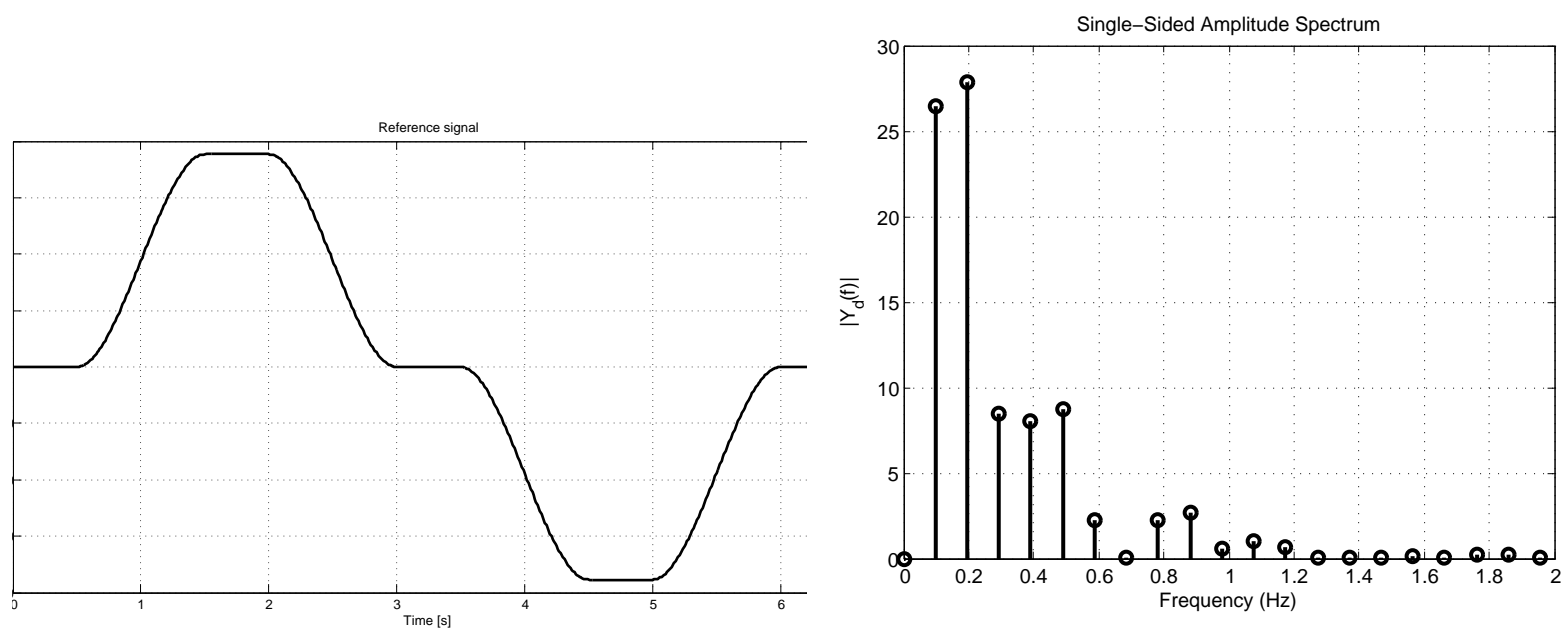

Figure 3: The reference trajectory (a) and its corresponding frequency spectrum (b).

equal or larger than $2 \mathrm{~Hz}$. In the results given below the $Q$-filter is chosen as a six order Butterworth 
filter, which is applied using the Matlab function filtfilt, to give a zero phase behaviour and the cut-off frequency of $2 \mathrm{~Hz}$. Also 2 frequency ranges are chosen, i.e., from 0 to $1.2 \mathrm{~Hz}$ and from 1.2 to 2 $\mathrm{Hz}$, respectively, where the first covers the reference signal bandwidth and this range is of primary interest because these frequencies have the most affect on the convergence rate. The second range includes those frequencies where the plant model accurately fits actual plant response and hence the control is effective.

Executing the design procedure given in Section 3.1 gives the feedback and learning controllers as

$$
\begin{gathered}
C=\frac{0.9582 z^{3}+0.7857 z^{2}-0.168 z-0.04756}{z^{3}-0.01955 z^{2}+0.005592 z+0.01334}, \\
L=\frac{4.544 z^{3}+6.16 z^{2}-1.603 z-0.02429}{z^{3}-0.01955 z^{2}+0.005592 z+0.01334},
\end{gathered}
$$

where $\mu_{1}=\sqrt{(0.8)}$ (upper bound on $\left|M\left(\mathrm{e}^{\mathrm{j} \omega}\right)\right|$ in the first frequency range - see (32) and (33)) and $\mu_{2}=\sqrt{(0.95)}$ in the second range. The resulting plot of $\left|M\left(\mathrm{e}^{\mathrm{j} \omega}\right)\right|$ is given in Figure 4(a) and confirms that the design specifications are met.

The designed ILC algorithm was experimentally tested over 10 trials. On completion of each trial the corresponding root mean square (RMS) value of the tracking error was computed using

$$
\mathrm{RMS}=\sqrt{\frac{1}{N} \sum_{p=1}^{N} e(p)^{2}},
$$

where $N$ is the number of sampled data in the trial time. In Figure 4(b) the RMS value of the tracking error is shown as a function of the trial number. As the result, this last figure confirms convergence of

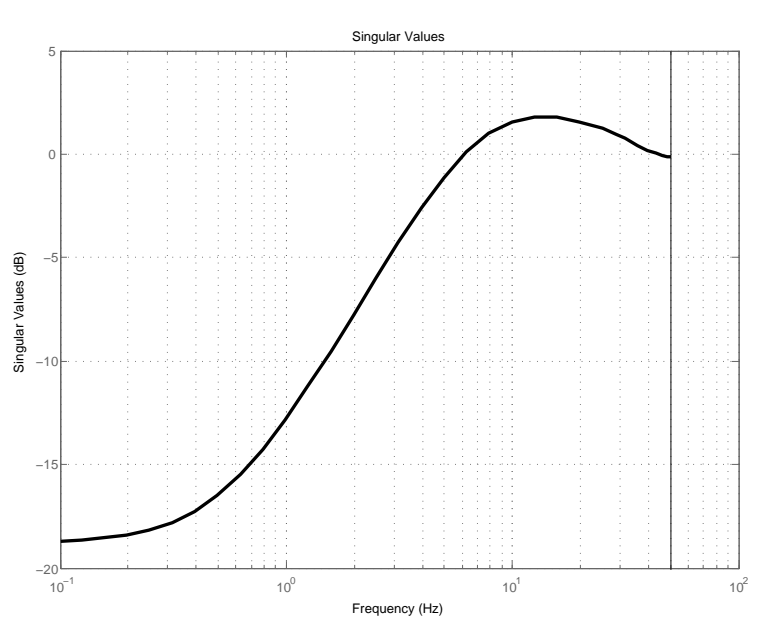

Figure 4: (a) Plot of $\left|M\left(\mathrm{e}^{\mathrm{j} \omega}\right)\right|$.

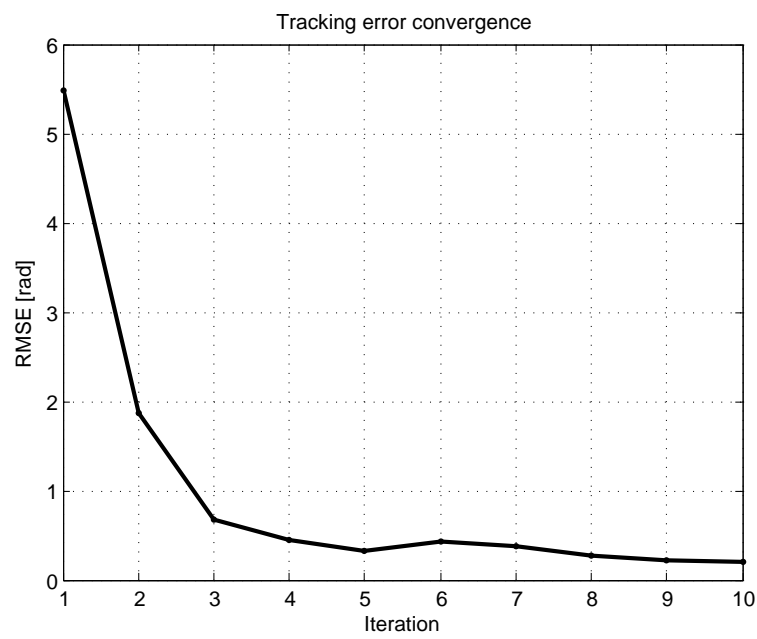

(b) The error convergence.

the error. To assess tracking performance, Figure 5 shows the RMS value of the tracking error plotted against trial number and the time axis. Obviously, the largest error occurred at the time instants when the system accelerated or decelerated. This corresponds to the low frequency behavior of the system. This plot demonstrates the good tracking performance of the new ILC design developed in this paper. In application implementation in discrete-time is attractive since ILC explicitly requires the storage of the previous trial data, which is typically sampled. Therefore, this implementation has first constructed a discrete model of the dynamics, designed the ILC controller using this model and proceeded to implementation using a micro-controller. Moreover, the sampling and design has 


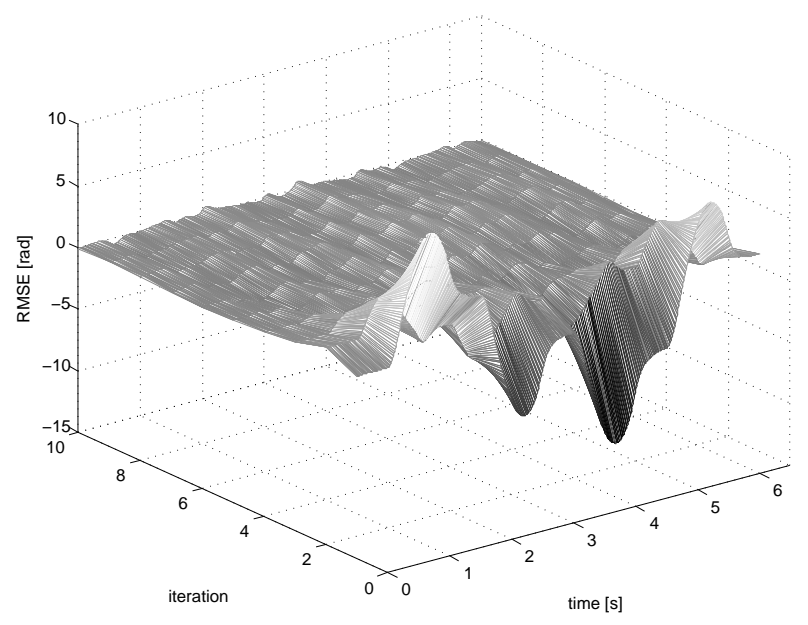

Figure 5: Error

to be done once only and hence there is no need to produce a new discrete-time model prior to each trial. Even when some conditions are changed, the ILC controller will attempt to generate a suitable feedworward signal to compensate for the effects of these changes on performancee. This means the control scenario has some robustness to changes in the plant parameters. For instance, see Figure 4(b), during 6th trial an unexpected change in the input signal generated by A/D converter occurred for a short period of time but this only caused a small increase in the tracking error and the design has some level of robustness to some (e.g., slow) parameter changes and further research on developing a robust version of this design should be undertaken.

\section{Conclusions}

In this paper a systematic procedure for the design of ILC laws using the repetitive process setting has been developed. Using this design it is possible to avoid adding weighting filters to obtain the required frequency response matrix to meet differing performance specifications. The results have been established by using the generalized KYP lemma to transform frequency domain inequalities over finite and/or semi-finite frequency ranges for a transfer-function to LMIs. Experimental validation of the results on a laboratory scale testbed have also been given.

This work has followed the majority of the ILC literature in assuming that all exogenous signals, i.e., reference signal and disturbances, are identical from trial-to-trial and that the initial conditions are the same for each trial and if these assumptions are violated then the achievable performance of the ILC design can be limited. If the initial state is not reset to the same vector on each trial, i.e., there are initial state shifts, the result could be additional transients which propagate across the trials and hence increase the tracking error. In many cases, non-repetitive disturbances caused by reset errors are located at high frequency rage (i.e., above the bandwidth of the reference signal) with repetitive disturbances assumed to be completely located in the low frequency range. It is therefore possible that the design in this paper can be developed further to remove a large part of non-repetitive disturbances since $Q$-filter attenuates high frequency disturbances (see Remark 1). Also this design should extend to the case when more feedback control effort is directed to eliminating the reset errors. One possible approach would be to impose additional constraints (bounds) on sensitivity function to make the feedback control part more effective and hence limit the effects of non-repetitive signals on performance of whole control system (feedback+ILC). This is an area for possible future research, for which the ideas in, e.g., Helfrich et al. (2010) are relevant. Also robust control should be investigated. 
The design of ILC for linear systems with higher relative degree has been the subject of considerable attention, where the results currently available in literature are based on the assumption that the learning controller is a static gain and the first Markov parameter of controlled system (i.e., $C B$ from the state-space triple $\{A ; B ; C\}$ ) defining the system model is known. However, the first Markov parameter is the response at time step one for a unit pulse input at step zero and hence, in typical situations, this parameter has zero (or near to zero) value. Therefore the solution can generate a very high gain and can result in very poor performance and transient dynamics. An alternative solution should include the fact that the desired trajectory must start from the first time step for which an input at zero can influence the output. This paper has developed a systematic way to design both learning and feedback controllers for systems with higher relative degree. It is shown that application of anticipative control law is possible and some transformations leads to a problem form where not all matrices of the resulting state-space model are changed.

One alternative way of designing ILC laws for strictly proper systems is given in Hładowski et al. (2011) but this design is based on state vector augmentation. Also, the dimensions of the matrix variables grow as the relative degree of the system increases. As a result, the controller order is also increased and can be greater than that of the system itself. The approach developed in this paper avoid these problems and the controller order does not increase with the plant relative degree. Also the size of matrix variables does not increase and therefore reduces the computational load when compared to the approach in Hładowski et al. (2011). Another approach was given in Paszke et al. (2012) where a low-pass filter with unity DC gain and sufficiently high cut-off frequency was used to remove a system limitation for the relative degree one case only. However, in practise it is hard to determine sufficiently high cut-off frequency prior to design procedure and also with choosing the order of the filter. Finally, this approach is applied when simple structure static learning and feedback controllers are used. These are significant limiting factors in the application of this approach that are overcome in this paper.

\section{References}

Ahn, H.S., Chen, Y.Q., and Moore, K.L. (2007), "Iterative learning control: brief survey and categorization," IEEE Transactions on Systems, Man and Cybernetics, Part C: Applications and Reviews, vol. 37, no 6, pp. 1109-1121.

Arimoto, S., Kawamura, S., and Miyazaki, F. (1984), "Bettering operation of robots by learning," Journal of Robotic Systems, 1, 123-140.

Barton, K., Mishra, S., Alleyene, A., Ferreira, P., and Rogers, J. (2011), "Control of high resolution electrohydrodynamic jet printing", Control Engineering Practice, vol. 19, no 11, pp. 1266-1273.

Bolder, J.J. (2015), "Flexability and robustness in iterative learning control with application to industrial printers," PhD Thesis, Eindhoven University of Technology, The Netherlands.

Bristow, D.A., Tharayil, M., and Alleyne, A. (2006), "A survey of Iterative Learning Control," IEEE Control Systems Magazine, vol. 26, no 3, pp. 96-114.

de Roover, D. (1996), "Synthesis of a robust iterative learning controller using an $\mathcal{H}_{\infty}$ approach," in Proc. 35th IEEE Conf. Decision and Control (CDC), 11-13 December, Kobe, Japan, pp. 30443049 .

de Oliviera, M.C., Bernussou, J., and Geromel, J.C. (2002), "Extended $\mathcal{H}_{2}$ and $\mathcal{H}_{\infty}$ norm characterizations and controller parametrizations for discrete-time systems," International Journal of Control, vol. 75, no 9, pp. 666-679. 
Doh, T.Y., and Ryoo, J.R. (2008), "Feedback-based iterative learning control for MIMO LTI systems," International Journal of Control, Automation, and Systems, vol. 6, no 2, pp. 269-277.

Freeman, C. T., Hughes, A.-M., Burridge, J. H., Chappell, P. H., Lewin, P. L., Rogers, E. (2009), "Iterative learning control of FES applied to the upper extremity for rehabilitation," Control Engineering Practice, vol. 17, no 3, pp. 368-381.

Freeman, C. T., Rogers, E., Hughes, A.-H., Burridge, J. H., Meadmore, K. L. (2012), "Iterative learning control in healthcare: Electrical stimulation and robotic-assisted upper limb stroke rehabilitation," IEEE Control Systems Magazine, vol. 32, no 1, pp. 18-43.

Gahinet, P., and Apkarian, P. (1994), "A linear matrix inequality approach to $\mathcal{H}_{\infty}$ control," International Journal of Robust and Nonlinear Control, vol. 4, no 4, pp. 421-448.

Heinzen, A., Gillella, P., and Sun, Z. (2011), "Iterative learning control of a fully flexible valve actuation system for non-throttled engine load control," Control Engineering Practice, vol. 19, no 12, pp. 1490-1505.

Heertjes, M., and Tso, T. (2007), "Nonlinear iterative learning control with applications to lithographic machinery," Control Engineering Practice, vol. 15, no 12, pp. 1545-1555.

Helfrich, B. E., Lee, C., Bristow, D. A., Xiao, X. H., Alleyene, A. G., Salapaka, S. M., and Ferreira, P. M. (2010), "Combined $\mathcal{H}_{\infty}$-feedback control and iterative learning control design with application to nanopositioning systems," IEEE Transactions on Control Systems Technology, vol. 18, no 2, pp. $336-351$.

Hładowski, L., Gałkowski, K., Cai, Z., Rogers, E., Freeman, C.T., and Lewin, P.L. (2010), " Experimentally supported 2D systems based iterative learning control law design for error convergence and performance," Control Engineering Practice, vol. 18, no 4, pp. 339-348.

Hładowski, L. Gałkowski, K., Cai, Z., Rogers, E., Freeman, C. T. and Lewin, P. L. (2011), "A $2 \mathrm{D}$ systems approach to iterative learning control for discrete linear processes with zero Markov parameter", International Journal of Control, vol. 84, no. 7, pp. 1246-1262.

Hładowski, L., Gałkowski, K., Cai, Z., Rogers, E., Freeman, C.T., and Lewin, P.L. (2012), "Output information based iterative learning control law design with experimental verification," Journal of Dynamic Systems, Measurement and Control, 134, pp. 021012/1-021012/10.

Inaba, K., Wang, C.C., Tomizuka, M., and Packard, A. (2009), "Design of iterative learning controller based on frequency domain linear matrix inequality," in Proc. American Control Conference (ACC), 10-12 July, St. Louis, USA, pp. 246-251.

Iwasaki, T., and Hara, S. (2005), "Generalized KYP lemma: unified frequency domain inequalities with design applications," IEEE Transactions on Automatic Control, vol. 50, no 1, pp. 41-59.

Kurek, J.E., and Zaremba, M.B. (1993), "Iterative learning control synthesis based on 2-D system tyheory," IEEE Transactions on Automatic Control, vol. 38, no 1, pp. 121-125.

Longman, R.W. (2000), "Iterative learning control and repetitive control for engineering practice," International Journal of Control, vol. 73, no 10, pp. 930-954.

Norrlöf, M. (2002), "An adaptive iterative learning control algorithm with experiments on an industrial robot," IEEE Transactions on Robotics and Automation, vol. 18, no 2, pp. 245-251. 
Oliveira, M.C.D., Bernussou, J., and Geromel, J.C. (2002), "Extended $\mathcal{H}_{2}$ and $\mathcal{H}_{\infty}$ norm characterizations and controller parametrizations for discrete-time systems," International Journal of Control, vol. 75, no 9, pp. 666-679.

Parmar, G., Barton, K., Awtar, S. (2014), "Large dynamic range nanopositioning using iterative learning control," Precision Engineering, vol. 38, no 1, pp. 48-56.

Paszke, W. Merry, R. and van de Molengraft, R. (2007), "Iterative Learning Control by twodimensional system theory applied to a motion system," in Proc. American Control Conference (ACC), 9-13 July, New York, USA, pp. 5484-5489.

Paszke, W., Gałkowski, K. and Rogers, E. (2012) "Repetitive process based iterative learning control design using frequency domain analysis", Proc. IEEE International Conference on Control Applications (CCA), Dubrovnik, Croatia, 3-5 October, 2012, pp. 1479-1484.

Paszke, W., Rogers, E., Gałkowski, K. and Cai, Z. (2013), "Robust finite frequency range iterative learning control design and experimental verification," Control Engineering Practice, vol. 21, no 10, pp. 1310-1320.

Paszke, W., and Bachelier, O. (2013), "Robust control with finite frequency specification for uncertain discrete linear repetitive processes," Multidimensional Systems and Signal Processing, vol. 24, no 4, pp. $727-745$.

Pipeleers, G., and Vandenberghe, L. (2011), "Generalized KYP lemma with real data," IEEE Transactions on Automatic Control, vol. 56, no 12, pp. 2942-2946.

Rogers, E., Gałkowski, K., and Owens, D.H., Control Systems Theory and Applications for Linear Repetitive Processes, Vol. 349 of Lecture Notes in Control and Information Sciences, Berlin, Germany: Springer-Verlag (2007).

Skogestad, S., and Postlethwaite, I., Multivariable Feedback Control: Analysis and Design, 2nd ed., John Wiley \& Sons, Inc (2005).

Tayebi, A., Abdul, S., Zaremba, M.B., and Ye, Y. (2008), "Robust iterative learning control design: application to a robot manipulator," IEEE/ASME Transactions on mechatronics, vol. 13, no 5, pp. 608-613.

Wang, Y., Gao, F., and Doyle, F.J. (2009), "Survey on iterative learning control, repetitive control, and run-to-run control," Journal of Process Control, vol. 19, no 10, pp. 1589-1600.

Wang, D. (2000), "On D-type and P-type ILC designs and anticipatory approach," International Journal of Control, vol. 73, no 10, pp. 890-901. 